\title{
The Study for Accuracy Assessment of Free PSA Density for Prostate Cancer Detection in Male Patients with High PSA
}

\author{
Tangkaew N, Vanichakarn D, Noppakulsatit P* \\ Division of urology, Department of surgery, Thammasat university hospital, Thailand
}

\begin{abstract}
Background: Prostate cancer detection is currently based on serum PSA with a digital rectal examination which is neither specific nor sensitive, which caused many unnecessary prostate gland biopsies that are highly expensive and can result in unwanted complications. Serum free PSA increases with a larger prostate gland,yet declines with a gland that contains cancer cells, thus prompting the hypothesis that calculating the ratio of serum free PSA against prostate gland volume provides the so-called "free PSA density" which can be utilized to improve prostate cancer detection.

Methods: Male participants were deemed eligible if they are at risk of prostate cancer with a PSA level of 4-10 ng/dL and aged between 50-75 years. Serum PSA and serum free PSA were obtained concurrently, followed by transrectal ultrasonography for prostate volume calculation and biopsy of the gland. Also reported are the sensitivity, specificity, positive predictive value, negative predictive value, accuracy, and receiver operating characteristic(ROC) with area under the ROC curve(AUC) of serum PSA, \%free PSA ratio, free PSA density and PSA density. The AUC of the variables were compared with the free PSA density and reported.
\end{abstract}

Results: The free PSA density cut point values which provided the highest accuracy was $0.025 \mathrm{mg} / \mathrm{mL} / \mathrm{cc}$, which had $61.5 \%$ sensitivity and $67.25 \%$ specificity. The ROC results indicate that \%free PSA ratio had the best AUC at 0.86 . Free PSA density and PSA density have AUC at 0.65 and 0.61 , respectively. Meanwhile, serum PSA had the worst AUC of 0.54. The researchers also calculated different AUCs of other variables to free PSA density. Finally, the AUC of free PSA density was significantly better than the reference standard tool serum PSA ( $p=0.022)$.

Conclusions: Prostate cancer is an emerging cancer among elderly men. Frequent use of serum PSA as a screening tool allows earlier diagnosis of this cancer but with the expensive of unnecessary further investigations. Most novel and promising tools are too expensive to be used as a generalized screening tool. From this study, free PSA density may be a reasonable alternative tool for detection of prostate cancer.

Keywords: Free prostate specific antigen density, Free PSA density, Free PSAD, PSAD, Prostate cancer screening tool, Prostate cancer

\section{Introduction}

Prostate cancer is the most common male genitourinary cancer. ${ }^{1}$ Prostate cancer detection is currently based on serum PSA with a digital rectal examination which is neither specific nor sensitive. Lojanapiwat Bet al., ${ }^{2}$ studied the use of serum PSA for prostate cancer detection in Thailand, and reported that serum PSA at $4.0 \mathrm{ng} / \mathrm{dL}$ had a $98 \%$ sensitivity and $9.3 \%$ specificity, while a serum PSA at $10.0 \mathrm{ng} / \mathrm{dL}$ had a $81.5 \%$ sensitivity and $55.5 \%$ specificity, ${ }^{2}$ which caused many unnecessary prostate gland biopsies that are highly expensive and can result in unwanted complications. Various prostate cancer screening tools exist which offer promising screening result,including PCA3, Prostatic health index, 4Kscore, ConfirmDX, yet these are expensive solutions. ${ }^{3-5}$ Serum free PSA is an alternative and affordable prostate cancer detection tool. ${ }^{6}$ PSA density (PSAD)is useful for discriminating higher serum PSA from a larger gland or from cancer cell production. Serum free PSA also increases
Quick Response Code:

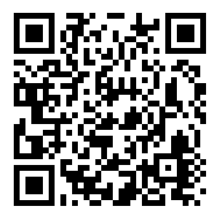

*Corresponding author: Pattranuch Noppakulsatit, Division of urology, Department of surgery, Thammasat university hospital, Pathumthani, Thailand

Received: 07 January, 2021

Published: 05 February, 2021

Citation: Tangkaew N, Vanichakarn D, Noppakulsatit P. The Study for Accuracy Assessment of Free PSA Density for Prostate Cancer Detection in Male Patients with High PSA. Trends Uro Nephro Res. 2021;1(1):1-5. DOI: 10.53902/TUNR.2021.01.000505 
with a larger prostate gland, yet declines with a gland that contains cancer cells, thus prompting the hypothesis that calculating the ratio of serum free PSA against prostate gland volume provides the so-called "free PSA density" which can be utilized to improve prostate cancer detection. ${ }^{1}$

\section{Method}

\section{Study oversight}

This study was conducted to assess accuracy of free PSA density for prostate cancer detection. The study was initiated in 2019 at Thammasat University Hospital, Thailand. Ethic committee approval was granted by Thammasat University Ethic Committee in Human Research (reference MTU-EC-SU-0-137/63).Data was collected by investigators. This study was not funded.

\section{Participants}

Male participants were deemed eligible for the present study if they are at risk of prostate cancer with a PSA level of 4-10ng/ $\mathrm{dL}$ and aged between 50-75 years. Serum PSA and serum free PSA were obtained concurrently, followed by transrectal ultrasonography (TRUS) for prostate volume calculation and biopsy of the gland. Tissues were sent to the Department of Pathology, Thammasat University Hospital, for histopathology interpretation. Patients were excluded if they had serum PSA or serum free PSA values and TRUS from another hospital, had evidence of urinary tract infection, had urinary retention and retaining of urethral catheter within the last 4 weeks, were using antiplatelet or anticoagulants which cannot be stopped for the operation and significant abnormal digital rectal examination defined as hard nodule, irregular surface, palpable seminal vesical or fixed to pelvic cavity.

\section{Test method}

The present study is a retrospective, cohort, diagnostic study that collected patient data from the first serum free PSA measurement undertaken at Thammasat University Hospital, Thailand between July 1, 2012 to May 1, 2020. If patients had multiple serum PSA and serum free PSA measurement samples, the most recent values before TRUS is used. Prostate gland volume was calculated by urologists following the equation: $0.52 \mathrm{x}$ transverse diameter $\mathrm{x}$ anteroposterior diameter $x$ cephalocaudal diameter equation. ${ }^{7}$ The biopsy was performed using the standard 12 extended cores random biopsy method. Patients were divided into two groups according to their histopathological results of the TRUS prostate gland biopsy, namely the benign prostatic hyperplasia (BPH) group and the prostate cancer group. Serum PSA, serum free PSA, \% free PSA ratio, PSA density, and free PSA density were collected, calculated,and reported for both groups.

\section{End points and assessments}

The primary end point was to determine the sensitivity and specificity of free PSA density for prostate cancer diagnosis. Also reported are the sensitivity, specificity, positive predictive value, negative predictive value, accuracy, and receiver operating characteristic(ROC) with area under the ROC curve(AUC) for each individual variable. The AUC of the variables were compared with the free PSA density and reported.

\section{Statistical analysis}

To assess the accuracy of free PSA density in prostate cancer detection, we needed patients with prostate cancer group for at least 32 based on power of 0.8 and alpha-error of 0.05 via STATA version 14.0. Sensitivity, specificity, positive predictive value, negative predictive value, accuracy and ROC was calculated and plotted. Student-T test was used for continuous data analysis.

\section{Results}

\section{Participants}

Between July 1, 2012 and May 1, 2020, a total of 136 males underwent all eligibility tests for inclusion in the study. Table 1 presents the baseline characteristics for all the male participants. A total of 12 individuals were excluded due to four abnormalities discovered during the digital rectal examination, three had urethral catheters, and a further five with a history of urinary retention within the last 4 weeks. No men were found to have a urinary tract infection or bleeding diathesis (Figure 1). The mean age of the patients was 66 years old,while the mean age of the prostate cancer group was slightly older at 71 years old. There were no differences in the use of alpha blocker and 5 alpha-reeducates inhibitors (5ARI) among the two groups.

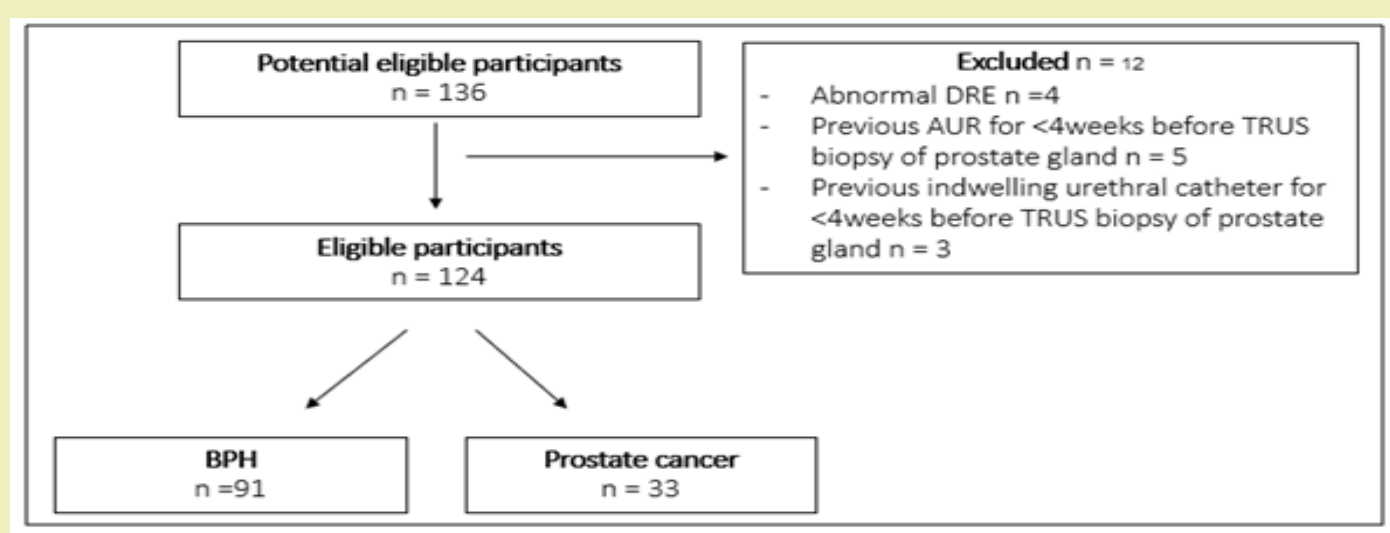

Figure 1: Trial profile. 
Table 1: Demographic and clinical characteristics of the patients.

\begin{tabular}{|c|c|c|c|}
\hline $\begin{array}{c}\text { Characteris- } \\
\text { tics }\end{array}$ & $\begin{array}{c}\text { Eligible patients } \\
\mathbf{n = 1 2 4}\end{array}$ & $\begin{array}{c}\mathbf{B P H} \\
\mathbf{n = 9 1}\end{array}$ & $\begin{array}{c}\text { Prostate cancer } \\
\mathbf{n = 3 3}\end{array}$ \\
\hline $\begin{array}{c}\text { Median age } \\
\text { (range) - yr }\end{array}$ & $66(50-75)$ & $65.5(50-75)$ & $71(50-75)$ \\
\hline $\begin{array}{c}\text { Alpha blocker - } \\
\text { No. (\%) }\end{array}$ & $83(79.0)$ & $63(78.75)$ & $20(80)$ \\
\hline 5-ARI - No. (\%) & $12(11.4)$ & $8(10)$ & $4(16)$ \\
\hline
\end{tabular}

\section{End points and outcomes}

No difference in the serum PSA values between the two groups were found, with a mean of $7.11 \mathrm{ng} / \mathrm{mL}$. Serum free PSA and \%freePSA ratio were significantly lower in the prostate cancer group com- pared to the BPH group at $0.956 \mathrm{ng} / \mathrm{mL}$ and $13.30 \%$ to $1.542 \mathrm{ng} /$ $\mathrm{mL}$ and $21.40 \%$,respectively ( $\mathrm{p}=<0.01$ for both variables). The BPH group did not have significantly larger glands than the prostate cancer group. PSA density was higher in the prostate cancer group at $0.211 \mathrm{ng} / \mathrm{mL} / \mathrm{cc}$ to $0.171 \mathrm{ng} / \mathrm{mL} / \mathrm{cc}$ for the BPH group. Free PSA density was significantly lower in the prostate cancer group compared to the BPH group at $0.0251 \mathrm{ng} / \mathrm{mL} / \mathrm{cc}$ and $0.0414 \mathrm{ng} / \mathrm{mL} /$ cc, respectively (Table 2). As the primary outcome, the free PSA density cut point values which provided the highest accuracy was $0.025 \mathrm{mg} / \mathrm{mL} / \mathrm{cc}$, which had $61.5 \%$ sensitivity and $67.25 \%$ specificity. Other variables cut point values are based on the international literature. The Positive predictive value, negative predictive value, andaccuracy of all the variables are presented in Table 3.

Table 2: Meanserum PSA, serum free PSA, \%freePSA ratio, PSAD, free PSAD.

\begin{tabular}{|c|c|c|c|c|}
\hline Characteristics & Eligible patients n=124 & BPH $\mathbf{n = 9 1}$ & Prostate cancer $\mathbf{n = 3 3}$ & P value \\
\hline Mean serum PSA+/-SD & $7.11+/-1.77$ & $7.05+/-1.75$ & $7.29+/-1.83$ & 0.498 \\
\hline Mean serum free PSA+/-SD & $1.402+/-0.690$ & $1.58+/-0.70$ & $0.944+/-0.351$ & $<0.001$ \\
\hline Mean free PSA ratio+/-SD & $19.71+/-7.70$ & $22.16+/-7.25$ & $12.96+/-3.99$ & $<0.001$ \\
\hline Mean PSA density+/-SD & $0.182+/-0.102$ & $0.171+/-0.095$ & $0.211+/-0.116$ & 0.056 \\
\hline Mean free PSA density+/-SD & $0.0348+/-0.0216$ & $0.0414+/-0.039$ & $0.0251+/-0.011$ & 0.006 \\
\hline Mean gland size+/-SD & $46.41+/-19.43$ & $48.61+/-20.30$ & $40.6+/-16.21$ & 0.072 \\
\hline
\end{tabular}

Table 3: Sensitivity, specificity, positive predictive value and negative predictive value ofPSA, free PSA ratio, PSAD, free PSAD.

\begin{tabular}{|c|c|c|c|c|c|c|}
\hline Characteristics & Cut point value & Sensitivity & Specificity & $\begin{array}{c}\text { Positive predictive } \\
\text { value }\end{array}$ & $\begin{array}{c}\text { Negative predictive } \\
\text { value }\end{array}$ & Accuracy \\
\hline Free PSA density & $0.025 \mathrm{ng} / \mathrm{mL} / \mathrm{cc}$ & $61.50 \%$ & $67.25 \%$ & 41.17 & $82.6 \%$ & $65.80 \%$ \\
\hline Serum PSA & $4.0 \mathrm{ng} / \mathrm{mL}$ & $100 \%$ & $0.01 \%$ & 11.1 & 72.1 & $55.20 \%$ \\
\hline$\%$ Free PSA ratio & $15.00 \%$ & $70.00 \%$ & $79.25 \%$ & $55.5 \%$ & $89.80 \%$ & $77.40 \%$ \\
\hline PSA density & $0.15 \mathrm{ng} / \mathrm{mL} / \mathrm{cc}$ & $60.60 \%$ & $52.70 \%$ & 20.9 & 67.7 & $60.40 \%$ \\
\hline
\end{tabular}

The ROC was plotted based on sensitivity and specificity. The results indicate that \%free PSA ratio had the best AUC at 0.86 . Free PSA density and PSA density have AUC at 0.65 and 0.61 , respectively. Meanwhile, Serum PSA had the worst AUC of 0.54. The researchers also Calculated different AUCs of other variables to free PSA density, which are shown in Figure 2 Finally, The AUC of free
PSA density was significantly better than the reference standard tool serum PSA $(p=0.022)$. Details of prostate gland biopsy results are depicted in Table 4. A significant proportion of patients in the Prostate cancer group had Gleason grade group 1 disease for $72 \%$ from all prostate cancer diagnosis.

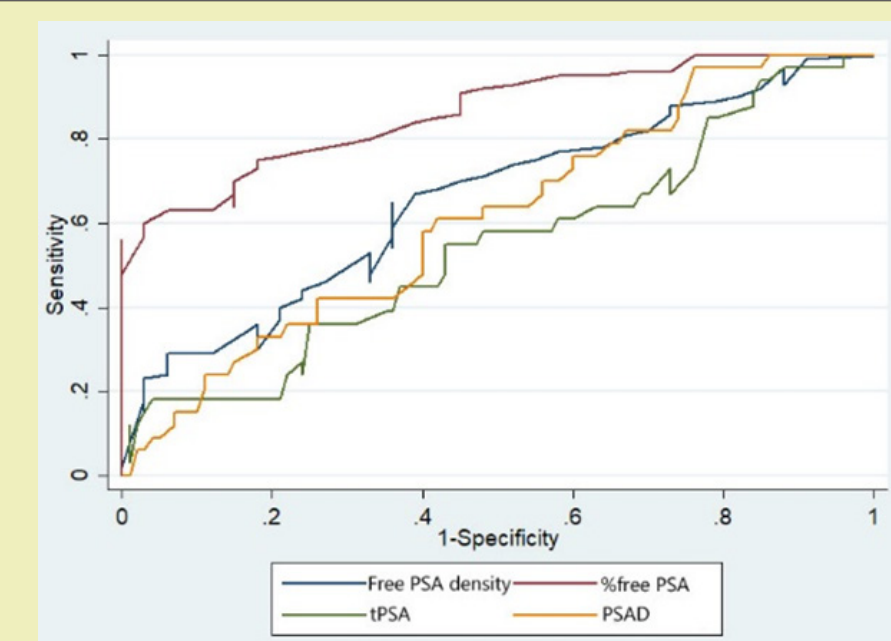

\begin{tabular}{|l|l|l|}
\hline & AUC & P value \\
\hline Serum PSA & 0.54 & 0.022 \\
\hline$\%$ free PSA ratio & 0.86 & $<0.001$ \\
\hline PSA density & 0.61 & 0.66 \\
\hline
\end{tabular}

Figure 2: Comparing AUC of free PSA density to other variables (free PSA density AUC $=0.65$ ). 
Table 4: Biopsy result of prostate cancer group.

\begin{tabular}{|c|c|c|}
\hline Characteristics & $\begin{array}{c}\text { Eligible patients } \\
\mathbf{n = 1 2 4}\end{array}$ & $\begin{array}{c}\text { Prostate cancer } \\
\text { group n=33 }\end{array}$ \\
\hline \multicolumn{3}{|c|}{ Gleason grade group } \\
\hline Gg 1 (3+3)-No. (\%) & $23(18.5)$ & $23(72)$ \\
\hline Gg 2 (3+4)-No. (\%) & $3(2.5)$ & $3(8)$ \\
\hline Gg 3 (4+3)-No. (\%) & $3(2.5)$ & $3(8)$ \\
\hline Gg 4 (8)-No. (\%) & $3(2.5)$ & $3(8)$ \\
\hline Gg 5 (9-10)-No. (\%) & $1(0.8)$ & $1(3)$ \\
\hline
\end{tabular}

\section{Discussion}

Prostate cancer is the most common male genitourinary cancer. ${ }^{1}$ Serum PSA remains the reference standard for prostate cancer screening among men aged between $50-75$ years. ${ }^{3}$ Men with serum PSA $2-10 \mathrm{ng} / \mathrm{mL}$ are at risk of developing cancer and may be used as a screening prompt. ${ }^{3}$ From international data, serum PSA at2.0$4.0 \mathrm{ng} / \mathrm{mL}$ had a sensitivity of $77-86 \%$ and specificity of $32-34 \%$ for cancer diagnosis, ${ }^{4,8}$ yet data from Thailand suggests that the cut point value of serum PSA should remain at $4.0 \mathrm{ng} / \mathrm{dL}$ for the Thai male population. ${ }^{2}$ Although a serum PSA cut point of $4.0 \mathrm{ng} / \mathrm{mL}$ is utilized in Thailand, a significant number of prostate gland biopsies are performed unnecessarily. ${ }^{3} \%$ free PSA ratio has gained attention due to its accuracy and cost-effectiveness. ${ }^{6}$ Since mutated cancer cells have malfunctioning free PSA synthesis, men with prostate cancer have significantly lower levels of serum free PSA.9., ${ }^{9,10}$ Aging men always experience prostate gland enlargement which can produce greater amounts of serum PSA than younger men. This results in high serum PSA resulting in a high proportion of false positive results among non-cancerous men. This makes PSA density-which is the proportion of serum PSA to prostate volume-useful to discriminate between higher serum PSA and BPH or from cancer cell production. $^{11}$

When the present study began, free PSA density had rarely been explored and limited data was available. Based on the above described theory, free PSA density may be an alternative screening tool for prostate cancer. ${ }^{1}$ This hypothesis prompted the researchers to conduct the present study. From Fu-Xaing Lin et al. study in 2017; free PSA density was used for prostate cancer screening in 303 men at risk. They reported sensitivity of $75.9 \%$ and specificity of $91.6 \%$ when use free PSA density cut point at $<0.033 \mathrm{ng} / \mathrm{mL} / \mathrm{cc}$. The study result shown that free PSA density sensitivity is even close to serum PSA but with much higher in specificity. ${ }^{1}$ According to the present study, free PSA density has better sensitivity, specificity, and AUC of ROC compared to the reference standard serum PSA. This makes free PSA density a reasonable, alternative prostate cancer screening tool. While the result of free PSA density is close to PSA density, it is still inferior to \%free PSA ratio. Furthermore, other studies achieved higher accuracy with free PSA density which could be due to some confounding factors described earlier. The main limitation of this study is the small population size, since all the male participants were required to undergo serum free PSA measurement, which is almost exclusively performed on men with previous equivocal serum PSA. Thus, this may not be appropriate for further generalized use as a screening tool. Yet due to data limitations with free PSA density, this study may act as a source of primary data for future investigations or trials. Another limitation is that some of the participants with definitive a high \%free PSA ratio-indicating a low risk for prostate cancer-did not undergo TRUS biopsy of the prostate gland and therefore not included in this trial.

\section{Conclusion}

Prostate cancer is an emerging cancer among elderly men. Frequent use of serum PSA as a screening tool allows earlier diagnosis of this cancer but with the expensive of unnecessary further investigations. Most novel and promising tools are too expensive to be used as a generalized screening tool. From this study, free PSA density may be a reasonable alternative tool for detection of prostate cancer and may have better results than serum PSA. Nonetheless, further clinical trials are required to clarify and refine the benefits of this tool.

\section{Acknowledgments}

None.

\section{Conflicts of interest}

None.

\section{Funding}

None.

\section{References}

1. Lin $\mathrm{F}-\mathrm{x}$. Free prostate specific antigen density predicts prostate cancer with high accuracy.Journal of biomedical research.2017;28(18):806-807.

2. Lojanapiwat B, Anutrakulchai W, Chongruksut W, et al. Correlation and diagnostic performance of the prostate-specific antigen level with the diagnosis, aggressiveness, and bone metastasis of prostate cancer in clinical practice. Prostate Int. 2014;2(3):133-139.

3. Carroll PR, Parsons JK, Andriole G, et al. NCCN Guidelines Insights: Prostate Cancer Early Detection, Version 1. 2018. J Natl ComprCancNetw. 2018;14(5):509-519.

4. Loeb S. Diagnosis and staging of prostate cancer. In: wein AJ, editor. Campbell-Walsh urology. 3. $11^{\text {th }}$ edition. Philadelphia: E; 2016. P. 2601-2608.

5. Narayan VM, Konety BR. Novel biomarkers for prostate cancer: An evidence-based review for use in clinical practice. Int $J$ Urol. 2017;24(5):352-360.

6. Bermúdez-Tamayo C, Martín Martín JJ, González Mdel P, et al. Costeffectiveness of percent free PSA for prostate cancer detection in men with a total PSA of 4-10ng/ml. Urol Int. 2007;79(4):336-344.

7. Baltaci S, Aksoy H, Turkolmez K, et al. Use of percent free prostatespecific antigen density to improve the specificity for detecting prostate cancer in patients with normal rectal examinations and intermediate prostate-specific antigen levels. Urol Int. 2003;70(1):36-41.

8. Xie GS, Lyv JX, Li G, et al. Prostate-specific Antigen Density Variation Rate as a Potential Guideline Parameter for Second Prostate Cancer Detection Biopsy. Chin Med J (Engl). 2016;129(15):1800-1804.

9. Woodhams S, Greenwell T. The clinical utility of measuring free-to-total prostate-specific antigen (PSA) ratio and PSA density in differentiating between benign prostatic hyperplasia and prostate cancer. Br J Urol. 1998;82(6):834-838. 
10. Moorgan TM. Prostate cancere tumor markers. In: Wein AJ, editor. Campbell-Walsh urology. 3. $11^{\text {th }}$ edition. Phladelphia: Elsevier; 2016. p. 2565-2578.
11. Zheng XY, Xie LP, Wang YY, et al. The use of prostate specific antigen (PSA) density in detecting prostate cancer in Chinese men with PSA levels of 4-10ng/mL. J Cancer Res Clin Oncol. 2008;134(11):1207-1210. 